(C) [2006] IEEE. Reprinted, with permission, from [Robert Steele, Chris Secombe and Wayne Brookes, Using Wireless Sensor Networks for Aged Care: The Patient's Perspective, Pervasive Health Conference and Workshops, 2006, Nov. 29 2006-Dec. 1 2006]. This material is posted here with permission of the IEEE. Such permission of the IEEE does not in any way imply IEEE endorsement of any of the University of Technology, Sydney's products or services. Internal or personal use of this material is permitted. However, permission to reprint/republish this material for advertising or promotional purposes or for creating new collective works for resale or redistribution must be obtained from the IEEE by writing to pubs-permissions@ieee.org. By choosing to view this document, you agree to all provisions of the copyright laws protecting it 


\title{
Using Wireless Sensor Networks for Aged Care: The Patient's Perspective
}

\author{
Robert Steele, Chris Secombe and Wayne Brookes
}

\begin{abstract}
This paper presents the findings of a qualitative study on the perceptions and thoughts of elderly people on the use of current sensor network technology for assisted aged care. Focus groups of elderly people were presented with examples of current sensor nodes and example scenarios of their use, and then invited to provide input on a range of issues surrounding the design and use of the technology. The focus group findings were verified with a health care professional as a control measure. This study examines sensing based interaction, implementation methodologies and user acceptance issues specifically for the elderly, and from the elderly's perspective. A significant finding of the study is that the two most important factors for elderly acceptance of sensor technology are cost and control.
\end{abstract}

Index Terms-Geriatrics, Health care, Patient monitoring, Wireless sensor networks

\section{INTRODUCTION}

$\mathrm{O}$ VER the last decade, there has been widespread recognition that a worldwide population shift will see effective and efficient aged care becoming an increasingly important issue, as advances in medicine and falls in morbidity rates result in an aging population.

Previous research has shown that as people grow older, they experience a steady decline in their cognitive, sensory and motor control functions [5], [8], [12], [21]. As [8] points out, these functional declines can result in tasks that seem simple to the young becoming difficult for the elderly; such as using a television remote control. Because of these functional declines, elderly people steadily become less able to look after themselves safely; and as a result, many elderly people are moved into retirement homes or other institutional care facilities. Approximately $5 \%$ of people over the age of 60 , and $20 \%$ of people over the age of 80 live in institutional care (Czaja, cited in [20], p.65). Studies have shown that many elderly people would prefer to live at home, but they cannot, as their ability to look after themselves safely is doubted.

Manuscript received July 3, 2006.

R. Steele is with the Faculty of Information Technology, University of Technology, Sydney, Australia (e-mail: rsteele@it.uts.edu.au).

C. Secombe, is with the Faculty of Information Technology, University of Technology, Sydney, Australia (e-mail: c.secombe@gmail.com).

W. Brookes is with the Faculty of Information Technology, University of Technology, Sydney, Australia (e-mail: brookes@it.uts.edu.au).
Wireless Sensor Networks are one theorised solution, which has significant promise in providing an effective, scalable product to respond to an increasing demand for aged care and elderly independence. These sensor networks consist of a collection of sensors, also known as motes, which can be used to monitor their surrounding environment and relay their readings onwards to provide useful aggregated information.

A significant amount of research has been conducted on various aspects in this field; however, most research seems to focus on the technology itself, rather than the patient's perceptions of the technology. For example, [7], [14], and [24] focus on the algorithms and computations involved in maintaining sensor network privacy, but the importance of privacy to a patient using sensor networks is not discussed or is not known.

The aim of this study is to provide information on what elderly people themselves perceive of current sensor network designs; opening up a channel of feedback between the technology designers and the intended users.

This study examines perspectives on the concept of sensing-based interaction and the challenges in designing sensor networks that are acceptable to the elderly. This study also investigates wearable sensor, embedded sensor and ambient monitoring implementation methodologies, and reports findings on elderly perceptions of sensor technology; by examining their perceived concerns. This is a sociotechnical study that brings together researchers' designs and elderly perceptions to form a view of the usability and acceptability of sensor networks for aged care.

\section{BACKGROUND}

The majority of sensor node technology is produced and sold by Crossbow Technology Incorporated, in their MICA, MICA2/MICA2DOT and MICAz range [4]. Intel Corporation are also developing sensor node hardware, but these are not available for commercial sale, and are only used in their own research laboratories [13]. Sensor nodes are currently quite expensive as they are a developing technology, with the newer nodes ranging from around \$USD100 per sensor node upwards [4]. Current sensor nodes are typically around the size of an Australian 10c coin, with a larger sized base station that they report back to, which can then relay information onwards to a computer network or call centre. 


\section{Methodology}

This study has used qualitative methodologies, including observation, interviews and focus groups. The centre of the study was a series of two focus groups. Focus groups were chosen as they allow for the free-flow of ideas and comments from participants, and minimise any impacts of observer influence on the group. The focus groups followed a semistructured design, with participants introduced to the concept of sensor networks for the elderly, and being given the chance to first raise issues that they foresaw on their own, and then any issues that had not been raised but had been identified in existing research were brought up and discussed.

Two focus group sessions were conducted, with the first being not only a forum of ideas, but also a pilot for the second focus group. The first, pilot focus group consisted of 5 elderly people, with all participants aged 65 or older. The gender distribution of this group was 3 females and 2 male participants. The group was presented with the concept of motes for aged care, and was shown an actual Crossbow MICA2DOT mote to give them an idea of what they looked like and current sizing of sensor nodes. They were given a chance to bring up issues that they foresaw on their own, and we also discussed the topics identified in existing literature to gain and document participant comments on issues perceived by researchers.

The second, main focus group was conducted two weeks after this first focus group. Ten participants were invited to participate in the second focus group; however only 8 participants arrived on the day. Of these 8 participants, the gender distribution was equal, with 4 men and 4 women present. Again, all participants were aged 65 or older. The focus group consisted of the same introduction to aged care and technology concepts, and the participants were also shown a Crossbow MICA2DOT mote for reference. The focus group was carried out in largely the same manner as the first group, except that the group dynamics were slightly different due to the larger group size. However, the larger number of participants allowed for a wider range of opinions.

Results from both focus groups were analysed, with key points noted and a study of the intersection of where the two groups agreed and where they disagreed. Common words, themes and concepts were grouped together for further analysis.

After analysis was complete and preliminary conclusions were drawn, the data and the findings were taken to a member of the health care profession for further validation. The health care professional was a carer from Hammond Care Group, who has been working in aged care for over 25 years and volunteered for this study. Each point from the data analysis was checked with her, to see whether or not she had observed the behaviour or preferences that the elderly had stated, in her professional carer career. This cross-validation was conducted in order to ensure that the elderly were being both truthful and realistic in their focus group responses.

\section{A. Study Limitations}

This study, through qualitative methods, provides strong indications, with considerable consistency and validity, of the thoughts, concerns and perceptions of the elderly on current sensor network designs. However, as this study does not use any quantitative or statistical measurements, no statistical significance can be drawn of the findings.

The results of this study are limited by several geographic and cultural factors. The study is limited to Australia - results may vary around the globe due to cultural factors, monetary factors and various states of the current public health system.

A significant limitation of this study is the fact that sensor network technologies are still being developed. Precise technology factors are unknown, such as the sizing of sensor nodes, battery size and life expectancy, as well as any costing and subsidisation. This study is limited to concepts - without exact details of the technology, a complete solution cannot be presented to the elderly, and as such, any answers received are based on the participants own assumptions of the technology. What the study does present, however, is feedback on each of the major theories and alternative methods of sensor network design and implementation currently being developed in the research community.

\section{STUDY FINDINGS}

Findings from the study are classified into two broad categories: Implementation Approach, and User Acceptance Issues. On the topic of implementation approach, three possible approaches were discussed in the focus groups, and opinions gathered:

- Wearable sensors;

- Ambient monitoring; and

- Embedded sensors.

On the topic of user acceptance, findings from the study are presented here in seven areas:

- Independence;

- Conceptual Difficulties;

- Cost;

- Self-Reliance and Dignity;

- Adherence;

- Social Isolation; and

- Privacy.

The following two sections present the findings in these areas in detail. Within each section, current research is examined first, followed by a summary and analysis of the findings from the focus groups and interview conducted.

\section{IMPLEMENTATION APPROACH FINDINGS}

One of the challenges in the field of health monitoring is to determine how sensor networks can best be deployed to serve the elderly. Balancing interface, usability, technology and cost considerations to determine the best method of implementing sensor networks in a home aged care situation 
is difficult. Ideally, the implementation allows the patient to maintain a full sense of independence whilst being minimally aware of the sensors' presence. There are currently three main approaches to implementing sensor networks for health monitoring: Wearable Sensors, Ambient Monitoring and Embedded Sensors.

\section{A. Wearable Sensors}

\section{1) Existing Research}

Wearable sensors are those which are typically embedded in clothing or clothing accessories, and are the size of a MICA2DOT mote or smaller. Ideally, wearable sensors are embedded in such a manner that they are not readily visible to the user. In a study conducted by Mikkonen, et. al, the participants themselves suggested the idea of using a wristband to provide security services in case of emergency, and stated that they would feel safer if they had a security wristband [18].

Pentland also carried out a study on wearable sensors, or "Smart Clothes" as it is termed in his research [23]. Pentland points out the benefits of using wearable sensors for medical applications, and claims that through the use of accelerometers and tilt sensors, sensors networks can distinguish between when a patient is sitting, standing and walking. Pentland also claims that biosensors can be used to indicate levels of mental arousal in a patient, although exactly what the term mental arousal describes is not defined. The author emphasises the value of having a mounted camera from the user's point of view, as it can approximately determine what the user is looking at.

Suzuki \& Doi conducted a study using wearable sensors to provide a portable health care monitoring system, titled "Lifeminder" [27]. The Lifeminder system was equipped with a pulse wave sensor, accelerometer, camera and microphone. According to Suzuki \& Doi, the pulse wave sensor detects changes in blood flow by using short wavelength irradiation of a section of the patient's skin, and a photo-diode to detect the reflected light. The camera was mounted to the pocket, and microphone to the patient's shirt. Suzuki \& Doi used the pulse wave sensor to detect when the user is stressed, as stress causes a significant change in pulse rate. Upon detection of a high stress situation, the sensor network would take a photo using the camera, and synchronise it with the voice recording to provide an overview of the situation at hand to an external observer.

\section{2) Findings \& Analysis}

The perceptions of the focus group participants of wearable sensors were mixed. Initial responses were that most participants don't mind the concept of wearing sensors in their clothes, but most were quick to point out perceived flaws, such as not wearing clothes in the shower when elderly are susceptible to falls, and simply forgetting to wear the sensor.

The idea of having wearable sensors as an accessory, in a watch, ring or necklace met with more enthusiasm. The sensor watch concept, proposed in Mikkonen et al's study [18] was the best received, with all participants concluding that it was the best of the wearable accessory options. However this approach is not flawless, as it limits what can be measured, and as some participants pointed out - not everyone wears their watch in the shower.

A few participants compared it to a hearing aid, which has beneficial outcomes, but it must be remembered, and that you simply may choose to wear it or not wear it depending on your mood that day. Participants pointed out that elderly people may not wear it simply because they want to rebel. Female participants also expressed concern about social implications of wearing a sensor. Male participants, whilst acknowledging the issue, did not find this to be as much of a significant concern. As one female participant commented, motes should be designed "so that everybody doesn't say 'Look at that woman, she's wearing one of those things'." This opinion is reinforced by the participants' comments on current panic alarm systems, which some deemed as too obtrusive, and therefore would refuse to wear them.

Overall, the general attitudes of the focus groups towards the idea of wearable sensors were quietly apprehensive. They indicated a willingness to wear them if they were demonstrated to be a practical solution, but wary of the fallacies of this method that they themselves perceived. Unless the problems of remembrance, rebellion and obtrusiveness can be overcome, this study would suggest that elderly people would resent the technology and not fully utilise this implementation method.

\section{B. Ambient Monitoring}

Ambient monitoring is a method which involves placing sensors around the environment to be monitored, rather than on the user themselves. In a home aged care scenario, this would typically involve placing numerous sensors throughout a house that track the actions of the user and detect emergency situations.

\section{1) Existing Research}

Mynatt et al. claim that ambient systems' implementations are more reliable than wearable panic-alarm systems such as Lifeline [15], as they do not require direct user action in the case of an emergency [20]. Mynatt et al. claim that the strengths of ambient implementations lie in the fact that they are less intrusive than wearable systems, and that the "out of sight, out of mind" maxim is highly valuable, to avoid an Orwellian "Big Brother" scenario [22].

Pentland also conducted a study using ambient monitoring [23], and points out that it can be hard to pinpoint a user's location without having a sensor on the body, as the human body takes various shapes depending on its stance and profile; the human body looks vastly different when lying down compared to standing up or bending over. As discussed above, this can be overcome to some extent in a wearable 
implementation with biosensors and tilt sensors; but in a strictly ambient implementation, the reliable detection of a human is much more difficult, especially without the use of a camera.

\section{2) Findings \& Analysis}

The concept of ambient monitoring met with slight indifference from the focus group participants. They expressed concerns about having sensors placed around their own home. The main concern that both groups raised, was that it would be more expensive than the other methods (wearable and embedded), as they imagined a larger number of sensors would be required to equip a whole house rather than a single person. It is unknown if this would be true in an actual implementation, but the logic has merit.

The participants were also quick to point out their perceived flaws in this approach - what happens when you go outside? Scenarios brought up in these focus groups included working in the garden, hanging out the laundry, or going shopping. However, participants from both groups also pointed out on their own accord, that generally speaking, if you are outside and you have an accident, someone will notice you or be able to hear you.

One group suggested that the use of both embedded and ambient methods would also be acceptable, and pointed out that a hybrid approach may solve a number of perceived problems.

Neither group were particularly concerned with the idea of "Big Brother" watching them. As one participant commented, "I don't care what it finds, if it saves my life". However, when presented with the idea of the sensor nodes also utilising a camera to verify situations, both groups unanimously rejected the idea, dismissing it as too intrusive.

\section{Embedded Sensors}

Embedded sensors are sensors which are typically embedded under the skin. While current mote technology is still too large to realistically implement this, it is envisaged that the sensor nodes, like all technology, will become smaller over time.

\section{1) Existing Research}

Existing research tends to treat the concept of embedded sensors as a sub-category of wearable sensors, as in a sense they are still being worn and face similar challenges. There is limited research directly in this area, but this area has several adjacent fields with similar concepts. Embedded sensors for aged care are similar in concept to RFID implant monitoring, which has already been deployed with limited success in some environments, such as border control and warehouse monitoring in Mexico [17]. However, the use of embedded technology to monitor a person's health has significantly different requirements to those of tracking warehouse stock.

2) Findings \& Analysis

The results here were somewhat surprising, as like other researchers, we had expected fierce resistance to this idea on the grounds of privacy and civil liberty. However, this was not the case. Both groups expressed a strong acceptance of the concept of embedding sensors under the skin.

Surprisingly, the initial comments of both groups were to compare the idea to the microchipping of dogs. Even though the concept of 'microchipping' humans has negative connotations, the participants expressed an acceptance of this idea. Their reasoning was that if it was embedded they wouldn't have to remember it or worry about it, and that no one would know they were wearing it.

Some participants were concerned that the embedded sensors might hurt to insert, and raised concerns about who would insert it for them. Both groups also had concerns about how would you know if the battery was running out (if the design uses a battery), and how the chip would be replaced in the event of a fault or battery change. Also, the participants enquired about how you would even know if the battery was running out, and if it had to be removed, would they have to 'cut it out'. The participants' main concerns were with the pain of insertion and removal, and maintenance of the battery and chip, rather than the concept of having a sensor embedded in their body.

When asked directly about whether or not they had any issues with the concept of being 'chipped', the answer was a fairly strong 'no' with both groups. As one participant commented on the issue, "When you get old you stop caring about that kind of stuff. If it's quick and painless and the benefits are there, I don't see any reason not to (be chipped)."

The elderly are typically considered as being daunted by new technology. However, participants from both groups indicated that they would not discriminate against sensor networks based on the fact that it's a new technology, as long as the value was obvious to them. The concept that elderly will readily accept technology but discriminate based on perceived value [12], appears to hold true for sensor networks. Simply put, as one participant commented, and all participants agreed "If the thing is good, and it works, then we go for it. However, if we see something that is useless, and obtrusive, and is change for change's sake, then no. Not interested.".

\section{USER ACCEPTANCE FINDINGS}

\section{A. Independence}

As the number of elderly people increases in future years, methods of enabling the elderly to stay at home will have an economic benefit, as well as allowing the elderly to maintain their highly valued independence.

\section{1) Existing Research}

Hanson et al. point out that independence in the aged care context can be hard to define, as it raises questions as to whether it is possible to truly have independence whilst living in monitored or assisted care [12]. It is unknown how independence, either true or relative, can be defined in such a 
situation; and if true independence is not possible, what facets of independence are most valuable.

Mynatt et al. identified three problem areas with elderly independent living: the ability to recognise a crisis, a lack of everyday cognition and detection of long term trends [20]. Mynatt et al. point out existing projects, such as Lifeline Medical Alarm [15] are helping, but still have unresolved problems as "Human-only solutions are fallible as humans are fallible". The authors' state that a number of problems arise with programs such as Lifeline which rely on user triggered events. For example, the elderly person may lack the ability to recognise a crisis, may forget to call or press an emergency button, or may be unconscious or have the emergency device out of reach.

\section{2) Findings \& Analysis}

The focus group participants in this study had varied responses to current "panic-alarm" projects like Lifeline. In Australia, a product known as VitalCall [3], provides similar functionality to Lifeline VitalCall involves the user wearing a neck or wrist pendant where they can push a button and be connected with a response centre. The participants generally had a fairly indifferent opinion of such systems, bordering on the negative side. One participant remarked about how her friend had one, and that her friend would not wear it outside as she was ashamed of it.

However, a few of the participants brought up a very good point - that at a base level, from the intended users' point of view, there is very little difference between using sensor networks for health monitoring and current panic alarm systems. The only significant difference apparent to the participants was that the user is potentially not aware of them, whereas panic alarms require an action, such as pushing a button. These points raised by the elderly participants tie in with Mynatt et al's failure scenario, wherein that the user, for whatever reason, may be unable to push the button [20].

However, the focus group participants indicated that the significance of this difference between the two systems to them is arguably not that great, and that it may make more sense to take the existing panic alarm system and refine it, rather than develop a new one. As one participant stated on the issue of not wearing panic alarms due to fear of being ostracised, "Well why don't they just make the panic alarms smaller?"

Whilst this simple comment does not take into account all the factors identified in [20] and the general research community, it can be said that existing panic alarms have the call centres, transmission protocols and infrastructure already in place as a starting point.

\section{B. Conceptual Difficulties}

\section{1) Existing Research}

Research has shown that the elderly have concerns regarding the use of technology which is relatively impersonal; to conduct what has largely been a social exercise. As Monk et al. point out, the elderly have expressed concerns about being "looked after by a robot" [19]. Mann also shares this view, as he states that widespread monitoring such as those needed for mobile solutions may be too intrusive; and "we might not want a world in which our every movement and conversation is being monitored by an external entity" [16]. Widespread monitoring, such as the smart spaces discussed in [16] raises concerns of an Orwellian society, in which every citizen is monitored to ensure they fit society's mould [22].

Mynatt et al's study points out that the solution to the conceptual problems may lie in developing a tool that is viewed by the elderly as a continuous partner rather than a tool [20]. However, as Lehto (cited in [18], p. 121) points out, that the solution may simply become more accepted in time, as today's working generation will be more accustomed to technology than the current elderly generation, and will have fewer negative attitudes towards technology.

\section{2) Findings \& Analysis}

The focus group participants did not express a strong concern about being monitored by an external entity, as long as a camera was not used. They did not have any objections to being monitored by sensors alone. Neither group saw sensor technology as a replacement to human care. As one participant pointed out, most people who opt for this technology probably wouldn't have an existing human carer as they are not fully incapacitated yet. Another participant commented that even if you did have an existing carer, they are not there all the time - this system could be used in conjunction with it to provide emergency assistance when the human carer is not present. Multiple participants commented that it would be advantageous to have both a sensor system and a human carer.

Other participants did not see the two topics as being connected. A few participants were of the opinion that this technology and human care are completely separate issues, as the sensor system is for emergencies only whilst carers are primarily there to bathe and feed people - something that sensors cannot do. Whilst all participants commented that it's not desirable to remove a source of human contact, they did not perceive the sensor system as directly affecting it; they believe that the sensor system and the human care are not interchangeable as they serve different purposes.

This assessment was confirmed by the health care professional, who does not see the two systems as being mutually exclusive. In fact, she pointed out that in her nursing home they use a motion detection system in conjunction with human care. The motion detection system notifies staff of any alarming or uncharacteristic behaviour which could possibly be an emergency, whilst the human carer still comes to provide meals and assistance as required. 


\section{Cost}

\section{1) Existing Research}

The cost of implementing and maintaining a sensor network is an issue that impacts on technology acceptance. In [18], the participants indicated they were willing to pay between 4-10 Euros per month for such a health service, but any increase above this price met with a sharp decline in acceptance rates. Only $14 \%$ of the elderly indicated that they were willing to pay more than 20 Euros a month for mobile healthcare in the form of a wristwatch [18]. As mentioned previously, a Crossbow mote costs upwards of USD $\$ 100$ per sensor node. A fully equipped node with the correct sensors for health care is probably more realistically around USD $\$ 250$ per node. This is a significant cost, and one that may place it out of reach of many elderly people.

Hanson et al. claim that the elderly control much of the wealth, ostensibly due to retirement and superannuation balances [12]. The acceptance of sensor technology in aged care relies partially on a balance between cost and benefits; as research has shown that the elderly are willing to pay for technology, but only if the value of it is demonstrated to them [12].

\section{2) Findings \& Analysis}

Cost was the most significant concern to the elderly participants in the focus groups. It was a frequently recurring theme and concern - even topics that were seemingly unrelated to the issue of cost were brought back to this central issue if a consensus could not be reached. Approximately half of the participants were receiving a government pension, and were all greatly concerned about the cost of implementing the system, and also the cost of running it and maintaining it. Both groups actually assumed that there would be an ongoing cost; it was not a concept that was presented to them.

All participants from both groups expressed a desire for it to be paid for or subsidised by someone else. Some participants suggested that it should be subsidised by the government. Others suggested that their family could pitch in and help with the cost of the system.

All participants saw this as an elective system, as they have choices and options about whether or not to use this system or to go into another alternative, such as nursed care. As they perceive this system as optional or elective, they were generally less willing to pay for it, but expressed a keen concern to use it if the cost was offset.

Both groups were quick to note that most elderly people are on a fixed income from either the pension or superannuation, and that could be a major limiting factor in regards to installation and ongoing costs. One participant commented that people on the pension in Australia get carers calling in to check on them for free, so that the willingness to pay for the system decreases sharply as they are already receiving free care from the government. One participant commented that with the current system, it also has the advantage of having human care rather than technological care - which they stated was not a major issue to them, but certainly a preference.

This cost-centric view of the focus group participants was also supported by the health care professional, who stated that in her experience, finance is the most significant concern for elderly people in their day to day lives. She also emphasised that the majority of people in her care are pensioners, and could not afford the care required if they had to pay for it.

\section{Self-Reliance \& Dignity}

\section{1) Existing Research}

Mynatt et al. point out that self-reliance and personal dignity are linked intricately, and that implementations must carefully balance between exerting partial control over someone's life, and assisting them [20]. When a system decides that it can make a better choice than the user, such as in the case of ambiguity discussed in [6], care must be taken to ensure that decisions made are completely correct. If the system perceives a situation incorrectly and recommends a course of action based on the incorrect reading, it can be perceived as an affront to the user's intelligence.

Abascal \& Civit [1] have a similar view to Mynatt et al, as they state that a device making a decision on behalf of a user results in a lack of personal autonomy, and can be regarded as an insult to the elderly person as it suggests that they lack the ability to make decisions.

\section{2) Findings \& Analysis}

The focus group participants perceived this issue as an extension of the central control issue. The participants gave mixed responses on whether or not a system should be able to override their decisions. Initially, both groups saw the concept as an affront to their intelligence, to the same extent found in [1] and [20].

The participants believed that as a whole, elderly people are generally reluctant to ask for help, but also believed that they have no problem asking for it when it's absolutely required. Anecdotal evidence may appear to go against this statement, as there is a somewhat common stereotype that elderly people will insist that they are fine even when they are not. Both groups were quick to point out that sensors are not as sophisticated as a human, especially a medically trained human, and expressed dubiousness over whether or not sensors will be able to correctly diagnose problems to the extent that they "know better than you do".

Both groups finally came to a consensus that yes, the technology should be able to override your decision, but only if it is absolutely sure. For example, if it detects that your heart stops beating (and is verified as not being a faulty sensor reading), then yes it should be able to send for help regardless of the elderly person's input. But this concept hinges on the premise that sensor networks will always correctly sense information, or be able to self-detect faulty readings and mark for re-sampling. 


\section{E. Adherence}

\section{1) Existing Research}

One of the biggest problems facing medicine and health care is non-adherence to medical regimens [9], [10], [25]. The average rate of adherence for patients taking medication is just 50\% [25], [26]. Therefore it can be said that a change to medical fields such as those introduced by sensor networks must be as minimal as possible; as any monitoring program that causes a level of distress to the user is less than $50 \%$ likely to be used correctly. In reality, as the concept involves the elderly rather than a 'typical' technology user, in the event of distress to the user requiring interaction, the real acceptance rate is probably well below $50 \%$.

Leventhal et al. (cited in [10], p. 18) states that there are two main patient reactions that determine the adherence rate of a medical recommendation. Gloyd classifies these two inputs as emotional context and cognitive context [10].

Gloyd defines emotional context as the manner in which medical information is presented to the user. This is traditionally determined by a patient-doctor report, or the doctors "bedside manner" [10]. The way in which a medical message is stated or written is critical in forming an emotional context, as a friendly, threatening or objective message will impact on the emotional context of the message, and therefore adherence. Gloyd points out that in digital applications, such as a sensor network, this is similar to the choice of warning sound used; the sound of a chime will convey a different emotional context to the sound of a buzzer [10].

The second determinant of adherence is cognitive context, which involves the patient's objective thoughts. The user will calculate their best choice based on the quality of the data presented. Gloyd uses the example of diabetes, where users monitoring their own blood sugar will make a cognitive decision based on their logical best course of action [10]. The author claims that clarity of the data is paramount to cognitive context. For example, a numeric output of the blood sugar level will result in the user calculating in their mind and adjusting their sugar, diet and exercise; whereas a simple message stating whether blood sugar is too high or too low is imprecise and does not convey the precise extent to which activities must be altered. Cognitive decisions are determined by the patient's mental calculation of the risks involved with the medicine, either by taking it or not taking it [10].

Gloyd states that careful interface design that addresses both the cognitive and emotional response levels of patients will result in positive user experiences, which produces higher adherence levels, and therefore higher acceptance levels [10]. Although, it can also be said that part of the lure of sensor networks is that the user does not have to adhere to a procedure; monitoring is automatic and does not require memorisation or calculation of medical recommendations.

2) Findings \& Analysis

The focus group participants saw the idea of remembering to wear a sensor node as a prominent issue, and were somewhat concerned that they may not adhere to the system by pure accident. The participants from both focus groups acknowledged the notion that elderly people often refused help and ignored medical advice, and both groups recited incidents from where they had observed this behaviour in their own lives.

However, the participants saw the concept of deliberately not adhering to the procedures as a mild concern, but also perceived this issue as an extension of the control issue. Opinions were split on the issue of whether or not the system should be able to be turned off. Some participants saw no valid reason for it to be able to be turned off and hence adherence was not a major factor, as the user is not required to remember anything (in a theorised embedded sensor situation).

Other participants staunchly desired the ability to turn the system off, even if they didn't use that particular functionality. These participants saw the ability to turn the system off as a form of control, and that if they chose to take the risks by turning it off, that it was still their choice to turn it off and take that risk.

Other participants saw a more moderated view, and suggested that the system should be able to be turned off in some instances, but if it is turned off, that it can only be turned off for a set amount of time, or that the system notifies the next of kin if the elderly person has turned it off, so that a family member may pursue an explanation of the elderly person as to why they have turned it off.

Whilst opinions were split and fiercely debated, the majority of participants were of the opinion that it should not be able to be turned off, and hence they don't have to remember to turn it on, and as there are no real valid reasons to switch the system off, then the functionality should not be offered. This view was backed up by the health care professional, who saw no valid reason for the system to be able to be turned off. She stated that in her opinion, although some elderly people may want the ability to turn it off, their families would not want them to have that functionality. She stated that in her experience, the elderly do often forget or disobey their medical recommendations. The health care professional emphasised the importance of the system being fully automated in order to avoid the adherence problem as much as possible.

\section{F. Social Isolation}

1) Existing Research

As people grow older, their social circles tend to diminish. Gregg [11] (citing Hummert et al. 1992) points out that significant links have been proven between quality and frequency of social interaction in older adults and their level of life satisfaction. Mikkonen et al's study [18] reinforces Gregg's findings, as they found that the elderly rank the maintenance of social relationships to be important; and have 
a desire for technology to assist them in achieving this goal. Abascal \& Civit also found that implementing mobile systems increases feelings of isolation, as it reduces human contact [1]. Additionally, the elderly do not typically wish to form new friendships outside of their social clubs and centres [2].

The implementation of sensor networks to monitor the elderly may contribute to a decline in social circles, as it removes an interaction between the patient and the caregiver. One of the advantages of using sensor networks to monitor the elderly is to enable them to maintain a level of independence and improve their life satisfaction. However, the implementation of sensor networks can reduce social contact, which as [11] shows, actually leads to the older person having a reduced life satisfaction. Hence, in a sense, the concept of using sensor networks to improve elderly life satisfaction appears fundamentally flawed.

Some researchers are attempting to accommodate for this perceived flaw by incorporating social aspects into sensor network design; although in different methods. Mynatt et al. propose using a history of sensor data to monitor activity levels [20], and several studies suggest using reminders or social suggestions if an elderly person has not contacted friends in a given timeframe [8], [11], [12].

Suzuki \& Doi emphasise the importance of medical monitoring for not only monitoring known conditions, but also in helping to prevent "lifestyle related disease" [27]. The authors point out that many ailments such as coronary disease and diabetes can be due to sedentary lifestyles.

\section{2) Findings \& Analysis}

The focus group participants viewed social wellbeing as an important aspect of their lives which significantly contributes towards their overall health. However, they did not see sensor networks, or any other technology as a threat of impacting on their social lives. All participants from both focus groups categorically denied that any sensor network system would impact on their social lives whatsoever.

The rationale of the elderly participants was that their social trends and habits would continue regardless of having the system or not. As one participant summed up, "If the elderly person was the kind of person who stayed inside and read books all day before, then they will continue to stay inside and read books regardless of whether or not they have this thing". All participants from both groups echoed this sentiment.

When the participants were presented with the ideas from existing research regarding the incorporation of social aspects into sensor network design, such as notifying family of a lack of activity, they were strongly opposed to the idea. Again, the majority of participants viewed this as an extension of the control issue. They saw it as their right to determine whether or not they go outside, and if so, how often, and strongly resented any attempt to dictate these terms to them.

At this point in both focus groups, the elderly reiterated their desire for the system to be purely for emergencies only.
They dismissed the social aspects completely, and stressed that the technology, in their opinion, should do one thing and one thing only - to call someone in the event of an emergency.

The health care professional thought the social isolation concept was relatively important, but also validated the point of view of the elderly. She stated that in her professional opinion, elderly people are often set in their ways and that an external influence, such as sensor networks, will not significantly impact on their social habits. However, she expressed a concern about removing the social contact from elderly people who have extremely limited social circles ( 1 or 2 people), as in her experience a visit from a carer can 'make an elderly persons day', and significantly impact on their wellbeing. She believed the removal of any social link for the elderly to be of significant concern, even if the elderly person is not aware of the significance, or if the elderly person denies the significance. However, she conceded that the extent of the impact largely depends on the individual's pre-existing social circles, as a person with an extremely small social circle will experience a much stronger impact than a person with wider social circles.

\section{G. Privacy}

\section{1) Existing Research}

Researchers typically believe that privacy in sensor networks for aged home care is of paramount importance, as the medical data being transmitted is confidential. Patients have a legal right to medical privacy, and fears for this are compounded when this information is transmitted through a readily accessible medium - the air. Abascal \& Civit point out that older users are traditionally concerned about privacy in wireless communication, as they feel that they cannot control the communication [1].

Challenges faced in providing sensor network privacy are differentiated from those facing typical wireless networks, due to their limited computing capacities. Threats to privacy can come from either passive attacks, such as listening in to data, or active attacks which involve manipulation of the data.

Elderly concerns for privacy are certainly founded, given the personal nature of the information transmitted. There is significant research into developing a realistic security protocol for sensor networks, with the most notable being SPINS [24] and TinySec [14]. These encryption protocols currently provide a sufficient level of security to ethically enable sensor network deployments with moderately sensitive data. However, it is important to note that providing data confidentiality on sensor networks has a high computational cost, leading to decreases in other important aspects such as battery life.

2) Findings \& Analysis

Contrary to popular belief amongst existing research, the focus group participants did not perceive privacy as a significant concern. Whilst they understood the concept of 
medical confidentiality, all participants stated that it was not important to them in this application - they did not perceive their medical data to hold any value.

None of the participants were significantly concerned when presented with the concept that someone could potentially 'listen in' to their data. One participant even remarked incredulously "What's in it that's private?" As a sensor network application in this field would only really carry medical information such as heart beat or pulse, then the importance of this data to the elderly is perhaps not as great as researchers have imagined; as little advantage can be gained by accessing it. As one participant pointed out, there are no obvious malicious activities that a person could potentially undertake with the knowledge of their heart beat. Some malicious applications have been theorised, such as the sensors being used to pinpoint an elderly person who could be seen as an 'easy target' and gaining location information in order to burgle them whilst they are in the shower. However, when presented with this potential for malicious intent, the elderly perceived it as trivial - "someone could get that information anyway, by looking in the window".

As one of the final questions, all participants from both groups were asked to rate their highest ranking and lowest ranking concerns out of all the issues that were discussed. In both groups, privacy was the lowest rating concern. The focus group participants also reiterated that a minority of older people may reject the technology on privacy grounds, but that the people who do strongly object are not being forced to use the system - it is purely elective. Another participant commented and met with widespread and emphatic agreement when she stated "when you get old you don't really care about privacy, you just want to know someone will come to help you".

When these findings were presented to the health care professional for validation, she agreed with the elderly participants' statements. In her experience, she stated that the majority of elderly people do not have significant concerns about the privacy of their medical information. She stated that a few people in her nursing home have asked general questions about the monitoring equipment and the information that they keep, but that none of the occupants have strongly objected to it. She also agreed with the elderly consensus that knowing that someone will come to help far outweighs the drawbacks of having medical information potentially available.

\section{SUMMARY OF FINDINGS}

\section{A. Conclusive Findings}

As this study encompasses a sizeable amount of data on varied aspects of sensor networks, there are numerous small conclusions that can be made about each aspect.

- The elderly see an embedded sensor methodology as preferable to ambient or wearable monitoring. Their main rationale for this decision is that they will not have to actively remember it, it has the potential to continue working outdoors, and other people will not know that they are wearing it. This view was validated by the health care professional.

- The elderly expressed a strong desire for some level of control over the system. The level of control may be limited, but they wanted to be able to feel some control over it, or to at least to have the illusion of control.

- In order to obtain the small level of control that the elderly desire, they must be able to interact with the sensor system in some fashion. This interaction method must be simple, such as the use of a single button or switch.

- The elderly believe that this technology should be for emergencies only. They strongly reject any attempt to incorporate social aspects into the technology. The participants believed that the system should have one function and one function only - to call for help in the event of an emergency.

- The elderly strongly rejected the use of any camera, video or still, in sensor network designs.

- The elderly did not perceive the technology as having any potential to change the breadth or quality of their social lives. They believed that how social a person is will remain constant, even if a link is removed between carer and patient.

- The elderly perceive cost to be the most significant factor in their acceptance and potential take-up of the technology. This was the most central and prominent issue to them, and they expressed a strong desire for the cost of the system to be offset by either family or government subsidisation.

- The elderly did not perceive privacy to be a significant concern. They believed their medical data to contain nothing of value to any malicious third party.

- Overall, the elderly perceive sensor networks as a workable solution to the current health care situation. Whilst they had concerns about some aspects of sensor network design, they could not identify or foresee any 'show-stopping' factors that would severely limit their acceptance of the technology, except for cost.

\section{B. Inconclusive / Interesting Findings}

This section summarises the aspects of sensor network technology the elderly participants could not reach a consensus on, and outlines other interesting side-issues that arose during the focus group sessions.

- The elderly were split in opinion on how to actually interact with the system, in order to gain the control they expressed a desire for. Some desired a screen, others beeping noises, others wanted a small device such as a watch with the status information on it. 
- Opinions were also split on what level of control the elderly should be able to have. Some participants thought full control, including the ability to turn the system on or off, whilst others thought minimal control was desirable, but just wanted to know that the option of control was there if they desired it.

- The elderly perceive few benefits of this system over existing products such as Lifeline and VitalCall. The only major benefit that they perceived was that they did not have to push anything to activate it. Some participants suggested that if current systems such as VitalCall could be improved, made smaller and less obtrusive, it may reduce the need for a sensor network system.

- Some nursing homes, such as the ones provided by Hammond Care Group, already utilise a form of sensor network technology. However, these are fixed wire and limited in their abilities (e.g. motion detection).

\section{CONCLUSION}

This study investigated the perceptions of the elderly on current sensor network designs. During the course of this study, as well as offering their own opinions on the technology, the elderly participants were asked about issues arising from existing research

However, regardless of the category of the question being asked, from the perspective of the focus group participants, the issue at hand often came back to one of two predominant factors - cost or control. These two factors appear to dictate and influence the majority of the elderly perceptions, and hence their acceptance of sensor network technology.

The focus group participants identified cost as their highest ranking concern surrounding the technology, and expressed a strong desire for subsidisation. Many participants stressed the fact that they are either on fixed and limited incomes, or on a pension, and as the usage of this technology would be elective, it does not rate highly on their financial agenda.

Control was also recurring theme among the elderly participants. Various levels of control were desired from different groups and individuals, but all participants expressed a desire for some level of control over the system. A lack of control was perceived to be somewhat an affront to their decision making ability and to be undermining their intelligence.

It is the primary finding of this study that the elderly perceive sensor networks as a viable solution to elderly care, on the condition that the two factors that govern elderly acceptance of sensor network technology, cost and control, are suitably addressed.

\section{REFERENCES}

[1] J. Abascal and A. Civit, "Universal access to mobile telephony as a way to enhance the autonomy of elderly people", Proc. EC/NSF Workshop on Universal Accessibility of Ubiquitous Computing, May 2001, pp. 93-99.
[2] C. Chen, M. Kobayashi, and L.M. Oh, "ShareComp: sharing for companionship", Proc. CHI 2005: Conference on Human Factors in Computing Systems, ACM Press, 2005, pp. 2074-2078.

[3] Chubb Security Inc (2005, Dec), VitalCall Personal Emergency Response Service, Chubb Security [Online]. Available: http://www.chubb.com.au/VitalCall.asp

[4] Crossbow Technology Inc (2005, Dec), Motes, Smart Dust Sensors, Wireless Sensor Networks [Online]. Available: http://www.xbow.com/Products/productsdetails.aspx?sid=3

[5] S. Czaja, Human factors research needs for an aging population, National Academy Press, 1990.

[6] A.K. Dey, D. Salber, G.D. Abowd, and M. Futukawa, "The conference assistant: combining context-awareness with wearable computing", Proc. 3rd International Symposium on Wearable Computers, IEEE Press, 1999.

[7] R.D. Di Pietro, L.V. Mancini, and A. Mei, "Random key-assignment for secure wireless sensor networks", Proc. 1st ACM Workshop on Security of Ad Hoc and Sensor Networks, ACM Press, 2003, pp. 62-71.

[8] J.L. Fozard, "Distribution of age and technology", ACM: Interactions, Mar 1997, pp. 42-43.

[9] W.D. Gentry, "Noncompliance to medical regimens", in Behavioural Approaches to Medical Treatment, Ballinger, 1997.

[10] D.M. Gloyd, "Positive user experience and medical adherence", Proc. 2003 International Conference on Designing Pleasurable Products and Interfaces, ACM Publications, 2003, pp. 17-21.

[11] J. Gregg, "Tearing down walls for the homebound elderly", Proc. CHI 2001: Conference on Human Factors in Computing Systems, ACM Press, 2001, pp. 469-470.

[12] V. Hanson, K. Coleman, M. Zaijcek, P. Gregor, J. Brown, M. Youssef, and R. Heller, "Design for dynamic diversity", Proc. EC/NSF Workshop on Universal Accessibility of Ubiquitous Computing: Providing for the Elderly Event Report, eds R. Heller, J. Jorge \& R. Guedj, ACM Publications, 2001.

[13] Intel Corporation (2005, Dec), Intel Mote: Sensor Nets / RFID [Online]. Available: http://www.intel.com/research/exploratory/motes.htm

[14] C. Karlof, N. Sastry, and D. Wagner, "TinySec: A link layer security architecture for wireless sensor networks", Proc. SenSys 2004, pp. 162175.

[15] Lifeline Systems Inc (2005, Dec), Lifeline - the most trusted medical alarm provider [Online]. Available: http://www.lifelinesys.com

[16] S. Mann, "Smart Clothing: Wearable multimedia computing and personal imaging to restore the technological balance between people and their environments", ACM Multimedia, 1996, pp.163-174.

[17] J. McHugh (2005, Dec), A chip in your shoulder: should I get an RFID implant?, Slate [Online], Available: http://www.slate.com/id/2109477

[18] M. Mikkonen, S. Vayrynen, V. Ikonen, and M.O. Heikkila, "User and concept studies as tools in developing mobile communication services for the elderly", ACM Personal and Ubiquitous Computing, vol. 6, no. 2, 2002, pp. 113-124.

[19] A. Monk, J. Brant, P. Wright, and J. Robinson, "CUHTec: the Centre for Usable Home Technology", Proc. CHI 2004 Conference on Human Factors in Computing Systems, ACM Publications, 2004, pp. 1073-1074.

[20] E.D. Mynatt, I. Essa, and W. Rogers, "Increasing the opportunities for aging in place", Proc. ACM Conference on Universal Usability, Nov 2000, pp. 65-71.

[21] V. Ogozalek, "A comparison of the use of text and multimedia interfaces to provide information to the elderly", Human Factors in Computing Systems, ACM Publications, 1994, pp. 65-71.

[22] G. Orwell, Nineteen eighty-four, Harmondsworth: Penguin, 1954.

[23] A. Pentland, "Perceptual intelligence", Communications of the ACM, vol. 43, no. 3, 2000, pp. 35-44.

[24] A. Perrig, R. Szewczyk, J.D. Tygar, V. Wen, and D.E. Culler, "SPINS: Security protocol for sensor networks", Wireless Networks 8, 2002, pp. 521-534.

[25] M.A. Rapoff, Adherence to Pediatric Medical Regimens, Kluwer Academic, 1999.

[26] D.L. Sackett and J.C. Snow, "The magnitude of compliance and noncompliance", Compliance in Healthcare, 1979, pp. 11-22.

[27] T. Suzuki, and M. Doi, "LifeMinder : An evidence-based wearable healthcare assistant", Proc. CHI 2001 Conference on Human Factors in Computing Systems, ACM Publications, 2001, pp. 127-128. 